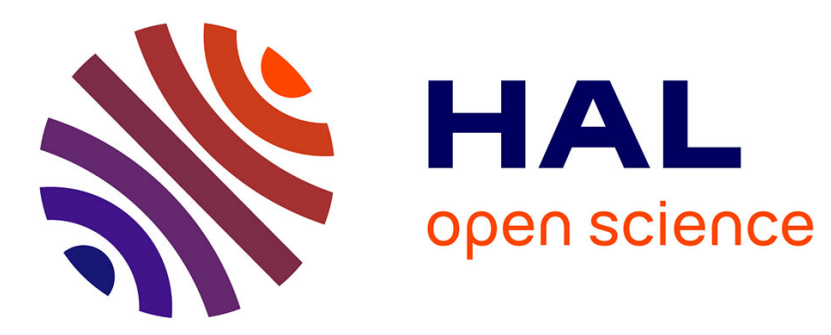

\title{
On the interface between two growing Eden clusters
}

Bernard Derrida, R Dickman

\section{To cite this version:}

Bernard Derrida, R Dickman. On the interface between two growing Eden clusters. Journal of Physics A: Mathematical and Theoretical, 1991, 24 (4), pp.L191-L193. 10.1088/0305-4470/24/4/006 . hal03285600

\section{HAL Id: hal-03285600 https://hal.science/hal-03285600}

Submitted on 19 Jul 2021

HAL is a multi-disciplinary open access archive for the deposit and dissemination of scientific research documents, whether they are published or not. The documents may come from teaching and research institutions in France or abroad, or from public or private research centers.
L'archive ouverte pluridisciplinaire HAL, est destinée au dépôt et à la diffusion de documents scientifiques de niveau recherche, publiés ou non, émanant des établissements d'enseignement et de recherche français ou étrangers, des laboratoires publics ou privés. 


\title{
LETTER TO THE EDITOR
}

\section{On the interface between two growing Eden clusters}

\author{
Bernard Derrida† and Ronald Dickman $\ddagger$ \\ † Service de Physique Theorique, CEN Saclay, 91191 Gif-sur-Yvette Cedex, France \\ $\$$ Department of Physics and Astronomy, Lehman College, City University of New York, \\ Bronx, NY 10468, USA
}

Received 11 December 1990

\begin{abstract}
We have measured the roughening exponent $\chi$ at the interface between a pair of growing Eden clusters. Our numerical results suggest that $\chi$ differs from the corresponding exponent for a freely growing interface, and that it depends on the initial geometry of the clusters, which grow into a vacant sector with an angle $\theta$. For $\theta<180^{\circ}$ we find $\chi=\frac{1}{3}$, and our results are consistent with $\chi=\frac{2}{3}$ for $\theta=180^{\circ}$ and $\chi=1$ for $\theta>180^{\circ}$.
\end{abstract}

Recently there has been much interest in the roughening properties of the free interface of a growing medium (Viscek 1989, Krug and Spohn 1990). A number of models, including the Eden model (Eden 1958) and ballistic deposition (Family and Viscek 1985), have been proposed to describe the interface of growing structures in situations ranging from the growth of a film by deposition to the growth of bacterial colonies or of tumours. A key quantity of interest is the roughening exponent $\chi$ which describes the fluctuations in local height $h(x)$ through $\left\langle[h(x)-h(y)]^{2}>\propto|x-y|^{2 x}\right.$. Numerical stimulations (Krug and Spohn 1990) of such models indicate that the roughening exponent $\chi$ is universal and depends only on the dimension $d$ of space (provided that the number or the size of the particles deposited in each growth step has a distribution which is not too broad (Zhang 1990)). For $d>2$ there is as yet no theory which yields the exact value of $\chi$ but the results of numerical simulations (Family and Viscek 1985 , Wolf and Kertesz 1987a, b, Kim and Kosterlitz 1989, Forrest and Tang 1990) and of Flory-like theories (Halpin-Healy 1989) predict that $\chi$ decreases with $d$. For $d=2$ the value $\chi=\frac{1}{2}$ has been obtained for some exactly soluble models (Meakin et al 1986), as well as from the theory of the Burgers' equation (Foster et al 1977, Huse et al 1985), and this prediction has been verified in many numerical studies (Wolf and Kertesz 1987a, b, Kim and Kosterlitz 1989).

In contrast with the free interface problem, the interface between two growing clusters has not been studied extensively. The purpose of the present work is to describe simulations of this process for a pair of Eden clusters, focusing on the roughening properties of the interface between them. Our results indicate that the interface is characterized by a roughening exponent $\chi$ which is different from the exponent $\chi_{\text {free }}$ for a freely growing interface. We also find that $\chi$ depends on the initial geometry of the clusters.

Our simulations follow the growth of a pair of clusters on the square lattice $(d=2)$, starting from a corner with an angle $\theta$, as depicted in figure $1(a)$. Clusters 1 and 2 grow into the semi-infinite region of initially empty sites, according to the Eden growth rule. Each site is described by an occupation index $\sigma_{i}$ which takes values 0,1 or 2 , 

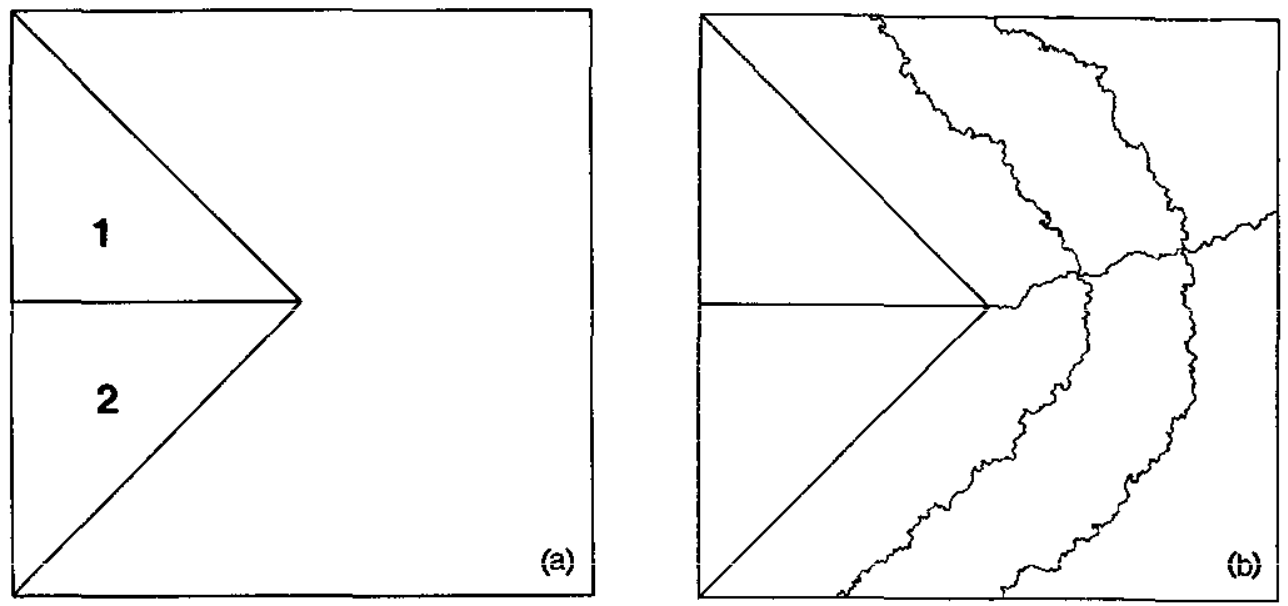

Figure 1. (a) The initial configuration for a pair of clusters which meet at an angle $\theta=270^{\circ}$. Sites in region 1 (2) belong to cluster 1 (2) and the remaining sites are vacant. (b) An example of the interface between the clusters. Also shown are the cluster boundaries at two intermediate stages.

corresponding to site $i$ vacant, or belonging to cluster 1 or cluster 2 , respectively. A list of (vacant) sites at the cluster boundaries is maintained throughout the process. In each growth step one of these sites is chosen at random, and is declared to belong to the cluster on whose boundary it lies. In case it is in the intersection of the boundaries, the site is randomly assigned to a cluster. Once each site in the $L \times L$ simulation region belongs to a cluster, the interface between the two clusters (i.e., the boundary between regions having $\sigma_{i}=1$ and $\sigma_{i}=2$ ), is examined. An example of such an interface, between two clusters which initially formed an angle of $270^{\circ}$, is shown in figure $1(b)$, along with the cluster boundaries at two intermediate stages of growth.

In order to study the roughening properties of this interface, $M(=2000)$ independent realizations of the growth process were performed. For each site $i$ we compuuted

$$
m_{i}=M^{-1} \sum_{s=1}^{M} \sigma_{i}(s)
$$

where $\sigma_{i}(s)$ is the value of $\sigma_{i}$ at the end of realization $s$. We define the width $w(x)$ of the interface at absissa $x$ as

$$
w(x)=\sum_{i}\left(m_{i}-1\right)\left(2-m_{i}\right)
$$

where the sum is over all sites with absissa $x$. The chief contribution to the sum comes from sites $i$ for which the probability $p_{i}$ to belong to cluster 1 is neither very small nor very close to 1 . In fact it is easily seen that

$$
\left(\left(m_{i}-1\right)\left(2-m_{i}\right)\right\rangle=p_{i}\left(1-p_{i}\right) .
$$

Hence $w(x)$ measures the spread of the interface at $x$. We performed simulations for several geometries (the values of $\theta$ shown in figure 2), and for lattice sizes $50 \times 50$, $100 \times 100,200 \times 200$ and $400 \times 400$. The end of the initial interface between clusers 1 and 2 was always placed at the origin of the lattice. 

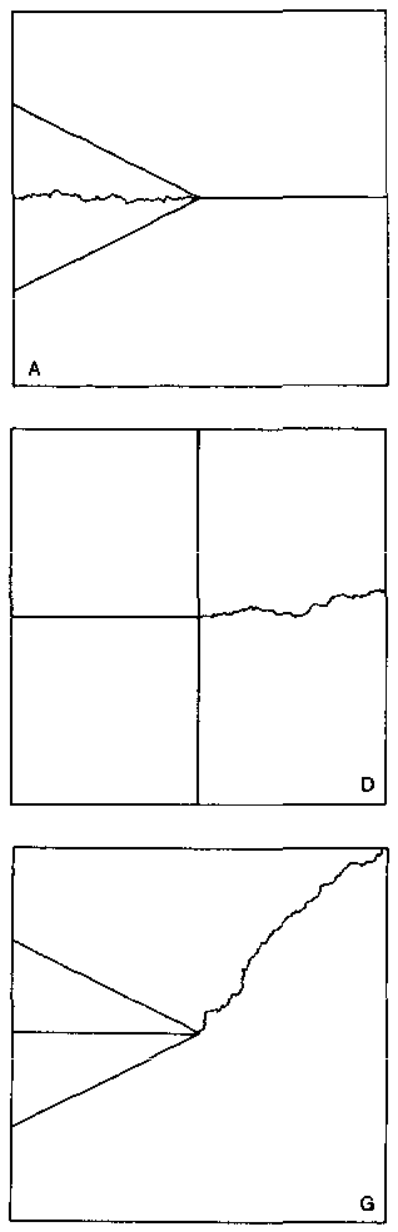
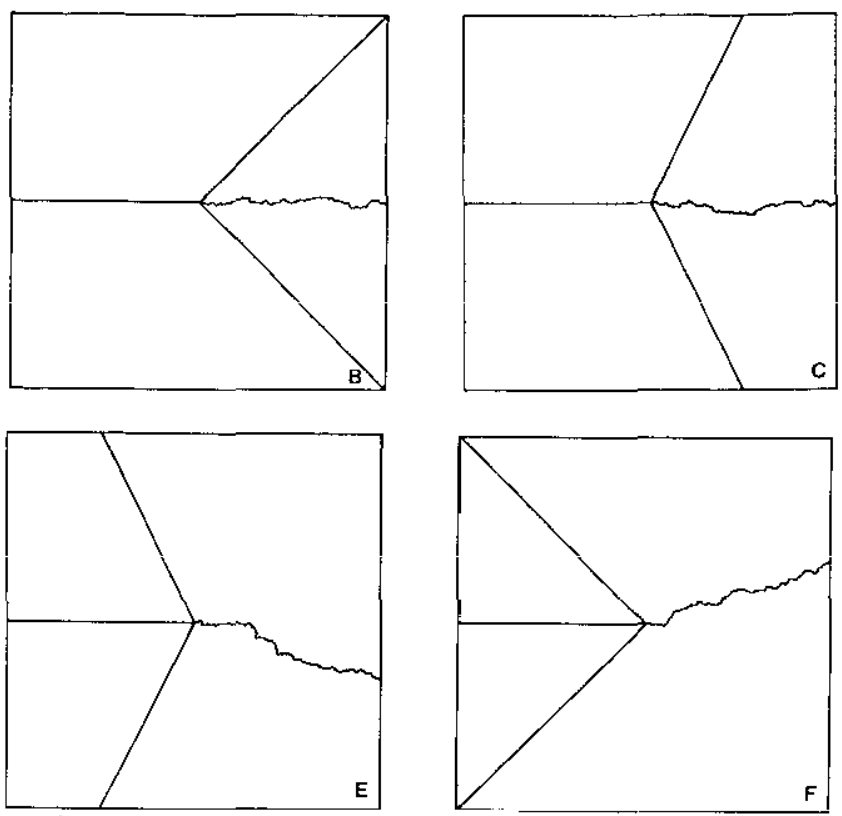

Figure 2. Examples of the seven growth geometries considered. The values for the initial angle $\theta$ are $\mathrm{A}: \tan (\theta / 2)=\frac{1}{2} ; \mathrm{B}: \tan (\theta / 2)=1 ; \mathrm{C}$ : $\tan (\theta / 2)=2 ; \mathrm{D}: \tan (\theta / 2)=\infty ; \mathrm{E}: \tan (\theta / 2)=-2 ; \mathrm{F}: \tan (\theta / 2)=-1$; $\mathrm{G}: \tan (\theta / 2)=-\frac{1}{2}$.

Our results (for $\ln w(x)$ against $\ln x$ ) are presented in figure 3 for the seven geometries considered, i.e. $\tan (\theta / 2)=\frac{1}{2}, 1,2, \infty,-2,-1$ and $-\frac{1}{2}$, corresponding to cases $A, B, \ldots, G$, respectively. Cases A, B and D exhibit well defined power-law behaviour in $w(x)$. In the other cases the graphs are somewhat curved and it is difficult to extract a reliable value for $\chi$. Moreover cases $\mathrm{F}$ and $\mathrm{G}$ show strong finite-size effects, in that the curves for different lattice sizes are not superimposed. On measuring the slopes in figure 3 for large values of $x$, one obtains the following estimates:

$$
\begin{aligned}
& \chi_{\mathrm{A}} \approx \chi_{\mathrm{B}} \approx 0.33 \quad \chi_{\mathrm{C}} \approx 0.39 \quad \chi_{\mathrm{D}} \approx 0.65 \\
& \chi_{\mathrm{E}} \approx \chi_{\mathrm{F}} \approx \chi_{\mathrm{G}} \approx 0.9 .
\end{aligned}
$$

However, because the graphs are curved in most cases, these exponent estimates should be regarded as preliminary; simulations on larger lattices may yield somewhat different values. We believe that our results are consistent with

$$
\chi= \begin{cases}\frac{1}{3} & \theta<180^{\circ} \\ \frac{2}{3} & \theta=180^{\circ} \\ 1 & \theta>180^{\circ} .\end{cases}
$$

At the moment we have no way, other than via simulations, to determine $\chi$, so the 

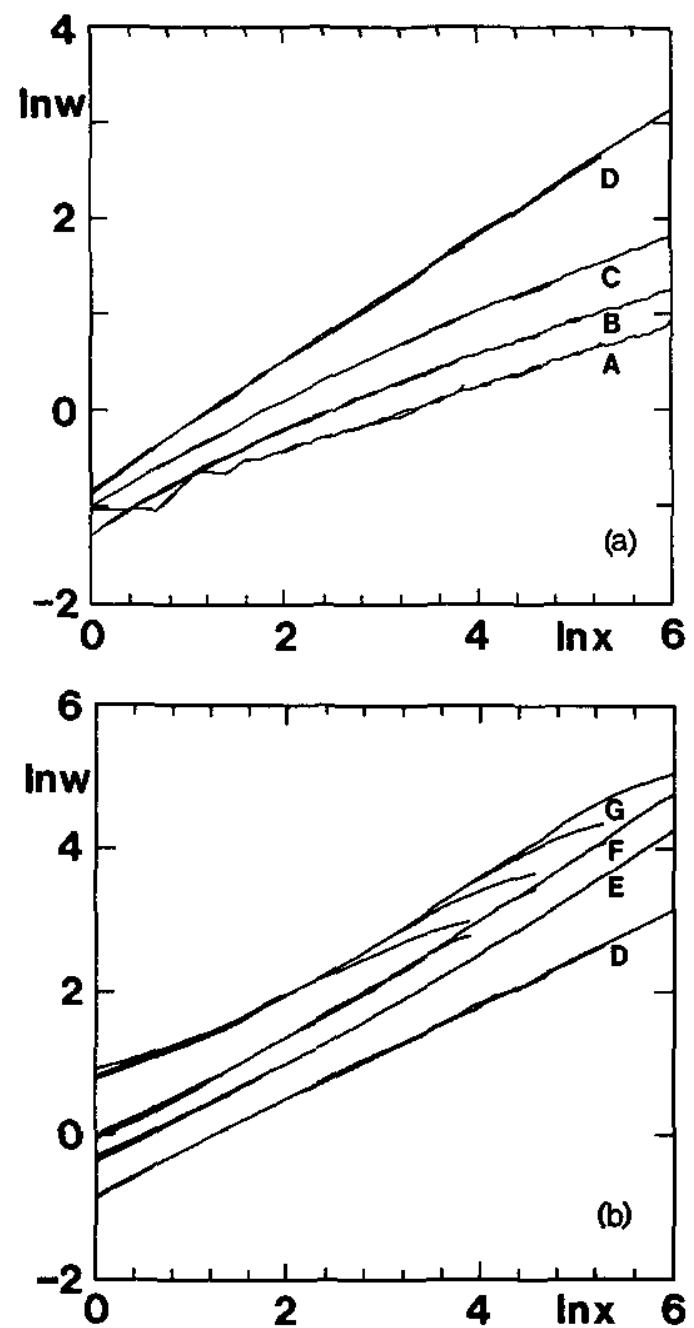

Figure 3. (a) Interface width $w(x)$ against $x$ for cases $A, B, C$ and D. (b) $w(x)$ against $x$ for cases D, E, F and $G$.

values proposed in (5) should be regarded as conjectures. (One can, however, imagine that a mean-field-like approximation of the kind recently developed to describe the roughening properties of an interface in the 2D Toom model (Derrida et al 1990) could be extended to the Eden interface problem to predict $\chi$.)

We also investigated several alternatives to the simulation procedure described above. In some cases the interface was initially oriented along the diagonal $y=x$, rather than along the $x$ axis. This geometry was used to study directed growth, in which clusters could only expand in the $+x$ and $+y$ directions. Finally, we considered a different definition of the position $Y(x)$, of the interface at $x: Y(x)=$ $\sup \left\{y \mid \sigma_{x, y} \neq \sigma_{x, y-1}\right\}$, (i.e. the first row at which one encounters the interface, starting from the top of a column). In this geometry a perfectly smooth interface would follow the line $y=x$, and so the width at $x$ may be defined through: $w^{2}(x)=\left\langle[Y(x)-x]^{2}\right\rangle$. None of these variations yielded any qualitative change in the interfacial roughening. 
The value $\chi=\frac{1}{3}$ for $\theta<180^{\circ}$ is supported by the following argument. One knows that for a single growing Eden cluster, with a flat interface at time $t=0$, the fluctuation of the height $h(t)$ at time $t$ is characterized by an exponent $\omega$, i.e.

$$
\left\langle h^{2}(t)\right\rangle-\langle h(t)\rangle^{2} \sim t^{2 \omega}
$$

with $\omega=\frac{1}{3}$ for $d=2$. The surfaces of a pair of clusters growing into a corner with $\theta<180^{\circ}$ grow freely until they meet. Therefore their height difference $h_{1}(t)-h_{2}(t)$ has fluctuations of order $t^{1 / 3}$ until they meet and the (local) growth process ceases. In effect, the interface is a record of the height fluctuation at the moment when the clusters meet. The time required for the cluster boundaries to meet at absissa $x$ is proportional to $x\left(t \simeq x / \sin (\theta / 2)\right.$. Therefore the difference in height $h_{1}-h_{2}$ typically has a magnitude $\sim x^{1 / 3}$, implying an interfacial width $w(x) \sim x^{1 / 3}$.

It would be interesting to study larger lattice sizes, in order to verify the conjectured values given in (5). As the shape of very large Eden clusters grown on the square lattice is known to be anisotropic (Krug and Spohn 1990) it is conceivable that for some lattices, or some other orientations, that the three regions we observed above still exist, but with a critical angle noticeably different from $180^{\circ}$. We also note that the measured value, $\chi \approx 0.9$ for $\theta>180^{\circ}$ (cases $\mathrm{E}, \mathrm{F}$ and $\mathrm{G}$ ), might not reflect the intrinsic roughness of the interface. In fact, the interfaces shown in figures $1(b)$ and $2 \mathrm{E}-2 \mathrm{G}$ do not appear much rougher than for $\theta=180^{\circ}$. Since the initiai angie between the clusters becomes rounded during the growth process, it is quite possible that asymptotically the roughening for $\theta>180^{\circ}$ is the same $\left(\chi=\frac{2}{3}\right)$ as for $\theta=180^{\circ}$. This is consistent with the results shown in figure $1(b)$ : the interface between the clusters is seen to be orthogonal to the cluster/vacuum boundary, which appears to flatten as the growth process continues. In this regard, we note that it may be necessary to revise the definition of the interface thickness $w(x)$ for $\theta>180^{\circ}$. If the interface were smooth, but had a random orientation, then we would find $\chi=1$, using $w(x)$ as defined above. Finally we note that it would also be of interest to change the geometry, for example by starting from a curved or a random interface.

\section{References}

Derrida B, Lebowitz J L, Speer E R and Spohn H 1990 to appear

Eden M 1958 Symposium on Information Theory in Biology ed H P Yockey (Oxford: Pergamon) p 359

Family F and Vicsek T 1985 J. Phys. A: Math. Gen. 18 L75

Forrest B M and Tang L H 1990 Phys. Rev. Lett. 641405

Foster D, Nelson D R and Stephen M J 1977 Phys. Rev. A 16732

Hâlpiñ-Healy T 1989 Fhys. Rev. Leti. 63917

Huse D A, Henley C L and Fisher D A 1985 Phys. Rev. B 552924

Kim J M and Kosterlitz J M 1989 Phys. Rev. Lett. 622571

Krug J and Spohn H 1990 Solids Far from Equilibrium: Growth, Morphology and Defects ed C Godrèche in press

Meakin P, Ball R, Ramanlal P and Sander L 1986 Phys. Rev. A 345091

Vicsek T 1989 Fractal Growth Phenomena (Singapore: World Scientific)

Wolf D E and Kertesz 1987a J. Phys. A: Math. Gen. 20257

- $1987 \mathrm{~b}$ Europhys. Lett 4651

Zhang Y-C 1990 J. Physique 512129 